\title{
Levels of Growth Differentiation Factor 15 and Early Mortality Risk Stratification in Cardiogenic Shock
}

\section{CardShock Investigators}

2019-11

CardShock Investigators, Hongisto , M , Kataja , A , Tarvasmäki , T , Holopainen , A , Javanainen , T , Jurkko , R , Jäntti , T , Kimmoun , A , Levy , B , Mebazaa , A , Pulkki , K , Sionis, A , Tolppanen , H , Wollert , K C , Harjola , V-P \& Lassus , J 2019 , ' Levels of Growth Differentiation Factor 15 and Early Mortality Risk Stratification in Cardiogenic Shock ' , Journal of Cardiac Failure , vol. 25 , no. 11 , pp. 894-901 . https://doi.org/10.1016/j.cardfail.2019.07.003

http://hdl.handle.net/10138/322098

https://doi.org/10.1016/j.cardfail.2019.07.003

publishedVersion

Downloaded from Helda, University of Helsinki institutional repository.

This is an electronic reprint of the original article.

This reprint may differ from the original in pagination and typographic detail.

Please cite the original version. 


\title{
Levels of Growth Differentiation Factor 15 and Early Mortality Risk Stratification in Cardiogenic Shock
}

\author{
MARI HONGISTO, MD ${ }^{1, *}$ ANU KATAJA, MD ${ }^{1, *}$ TUUKKA TARVASMÄKI, MD, PhD, ${ }^{2}$ ANU HOLOPAINEN, MSc ${ }^{3}$ \\ TUIJA JAVANAINEN, MD ${ }^{2}$ RAIJA JURKKO, MD, PhD, ${ }^{2}$ TONI JÄNTTI, MD, ${ }^{2}$ ANTOINE KIMMOUN, MD, PhD, ${ }^{4}$ \\ BRUNO LEVY, MD, PhD, ${ }^{5}$ ALEXANDRE MEBAZAA, MD, PhD, ${ }^{6}$ KARI PULKKI, MD, PhD, ${ }^{7}$ ALESSANDRO SIONIS, MD, ${ }^{8}$ \\ HELI TOLPPANEN, MD, PhD, ${ }^{2}$ KAI C. WOLLERT, MD,,${ }^{9,10}$ VELI-PEKKA HARJOLA, MD, PhD,${ }^{1}$ AND JOHAN LASSUS, MD, PhD ${ }^{2}$, \\ FOR THE CARDSHOCK INVESTIGATORS \\ Helsinki, Kuopio, and Turku, Finland; Nancy, and Paris, France; Barcelona, Spain; and Hannover, Germany
}

\begin{abstract}
Background: The aim of this study was to assess the levels, kinetics, and prognostic value of growth differentiation factor 15 (GDF-15) in cardiogenic shock (CS).

Methods and Results: Levels of GDF-15 were determined in serial plasma samples (0-120 h) from 177 CS patients in the CardShock study. Kinetics of GDF-15, its association with 90-day mortality, and incremental value for risk stratification were assessed. The median GDF-15 $5_{\text {oh }}$ level was 9647 ng/L (IQR 4500-19,270 $\mathrm{ng} / \mathrm{L})$ and levels above median were significantly associated with acidosis, hyperlactatemia, renal dysfunction, and higher 90 -day mortality $(56 \%$ vs $28 \%, P<.001)$. Serial sampling showed that non-survivors had significantly higher GDF-15 levels at all time points $(P<.001$ for all). Furthermore, non-survivors displayed increasing and survivors declining GDF-15 levels during the first days in CS. Higher levels of GDF-15 were independently associated with mortality. A GDF-15 $12 \mathrm{~h}$ cutoff $>7000 \mathrm{ng} / \mathrm{L}$ was identified as a strong predictor of death (OR 5.0; 95\% CI 1.9-3.8, $P=.002)$. Adding GDF-15 $12 \mathrm{~h}>7000 \mathrm{ng} / \mathrm{L}$ to the CardShock risk score improved discrimination and risk stratification for 90-day mortality.

Conclusions: GDF-15 levels are highly elevated in CS and associated with markers of systemic hypoperfusion and end-organ dysfunction. GDF-15 helps to discriminate survivors from non-survivors very early in CS. ( Cardiac Fail 2019;25:894-901)
\end{abstract}

Key Words: Cardiogenic shock, growth differentiation factor 15 (GDF-15), prognosis, biomarkers.

Cardiogenic shock (CS) is a state of emergency determined by severe systemic hypoperfusion due to cardiac dysfunction. Despite remarkable advances in the treatment of myocardial infarction and intensive care, mortality in CS remains unacceptably high. ${ }^{1,2}$ A systemic inflammatory response and multiorgan injury contribute to the high fatality rates in CS. Therapy options like advanced circulatory support are invasive, highly intense, and costly. Recently, clinical risk scores for predicting outcome have been put forward in CS. ${ }^{3,4}$ Biomarkers have shown good potential for prognostic risk stratification in cardiovascular disease and could eventually be helpful in classifying patients eligible for specific therapeutic strategies in CS. ${ }^{5,6}$

Growth differentiation factor 15 (GDF-15), a member of the transforming growth factor- $\beta$ cytokine superfamily, has emerged as a strong prognostic biomarker in cardiovascular

From the ${ }^{1}$ Emergency Medicine, University of Helsinki and Department of Emergency Medicine and Services, Helsinki University Hospital, Helsinki, Finland; ${ }^{2}$ Cardiology, Helsinki University and Heart and Lung Center, Helsinki University Hospital, Helsinki, Finland; ${ }^{3}$ Department of Clinical Chemistry, Institute of Clinical Medicine, University of Eastern Finland and ISLAB, Kuopio, Finland; ${ }^{4}$ Medical Intensive Care Unit Brabois, Institut Lorrain du Coeur et des Vaisseaux, CHRU de Nancy, INSERM U1116, Université de Lorraine, Nancy, France, ${ }^{5}$ Service de Réanimation Médicale Brabois, CHRU Nancy, Pôle Cardio-Médico-Chirurgical, 54511 Vandoeuvre-les-Nancy, INSERM U1116, Faculté de Médecine, 54511Vandoeuvre-les-Nancy and Université de Lorraine, Nancy, France; ${ }^{6}$ INSERM U942, APHP, Hôpitaux Universitaires Saint Louis Lariboisière and University Paris Diderot, Paris, France; ${ }^{7}$ Department of Clinical Chemistry, University of Turku and Turku University Hospital, Turku, Finland; ${ }^{8}$ Acute and Intensive Cardiac Care Unit, Cardiology Department, Hospital de la Santa Creu i Sant Pau, IIB Sant Pau, CIBER-CV, Universitat Autonoma de Barcelona, Spain; ${ }^{9}$ Department of Cardiology and Angiology, Hannover Medical School, Hannover, Germany and ${ }^{10}$ Division of Molecular and Translational Cardiology, Hannover Medical School, Hannover, Germany.

Manuscript received February 26, 2019; revised manuscript received June 9, 2019; revised manuscript accepted July 5, 2019.

Reprint requests: Johan Lassus, MD, PhD, Heart and Lung Center, Cardiology, Helsinki University Hospital: Jorvin sairaala, Turuntie, 150 POB 800 , 00029 HUS, Finland. Tel: +358 50 5765781, Fax: +358 9471 85922. E-mail: johan.lassus@ fimnet.fi

Funding: This study was financially supported by Aarne Koskelo Foundation and The Finnish Cardiac Foundation. Laboratory kits were provided by Roche Diagnostics.

*Equal contribution to the manuscript.

See page 900 for disclosure information.

$1071-9164 / \$$ - see front matter

(c) 2019 Elsevier Inc. All rights reserved.

https://doi.org/10.1016/j.cardfail.2019.07.003 
disease. ${ }^{7}$ GDF-15 is weakly expressed in most tissues under physiological circumstances but may be strongly induced in response to acute stressors including inflammation, oxidative stress, hypoxia, and tissue injury. ${ }^{7,8}$ GDF-15 has been shown to provide independent prognostic information beyond traditional clinical risk factors and established biomarkers in acute coronary syndromes (ACS), including ST-elevation myocardial infarction, and in heart failure. ${ }^{9-16}$ However, GDF-15 is not a cardiac-specific biomarker. In advanced heart failure, GDF-15 appears to be mainly derived from peripheral tissues reflecting systemic and extra cardiac pathologies. ${ }^{17}$ Stress-induced expression, through p53-mediated pathways, of GDF-15 in macrophages, vascular smooth muscle, and endothelial cells makes it a potential marker of vascular injury. ${ }^{5,6}$ Data on GDF-15 in critically ill patients are still scarce. Based on its association with systemic and vascular abnormalities, GDF-15 may be of particular interest in CS.

The aim of our study was to assess the levels of GDF-15 in CS using serial measurements and to analyze its prognostic properties and incremental value for risk stratification in CS.

\section{Methods}

The CardShock study (NCT01374867 at ClinicalTrials. gov) is a prospective, observational, multicenter study on CS. The overall aim of the CardShock study was to investigate the aetiology, clinical and biochemical characteristics, and to describe management and prognosis in contemporary CS. Specific aims were to identify novel prognostic risk markers in this medical emergency. Patients $(n=219)$ were recruited in 8 European countries at 9 tertiary hospitals between October 2010 and December 2012. A detailed description of the study population, treatments, and overall mortality has been previously published. ${ }^{3}$

\section{Inclusion Criteria and Data Collection}

Patients had to be $>18$ years old and enrolled within 6 hours from the identification of CS. In addition to an acute cardiac cause (both ACS and non-ACS patients were included), the inclusion criteria required systolic blood pressure to be $<90 \mathrm{mmHg}$ despite adequate fluid challenge or need for vasopressor therapy to maintain systolic blood pressure $>90 \mathrm{mmHg}$ and signs of hypoperfusion (altered mental status/confusion, cold periphery, oliguria $<0.5 \mathrm{~mL} / \mathrm{kg} / \mathrm{h}$ for the previous 6 hours, or blood lactate $>2 \mathrm{mmol} / \mathrm{L}$ ). Patients presenting with hemodynamically significant cardiac arrhythmia or shock after cardiac or non-cardiac surgery were excluded from the study. Baseline characteristics, medical history, and clinical findings were recorded at the time of detection of the shock. Biochemical and hemodynamic data as well as treatment and procedures were registered at baseline and until 120 hours after inclusion at prespecified time points. Patients were treated according to local clinical practice. Written informed consent was obtained from the patient or next of kin if the patients were unable to give the consent on admission. The study was approved by local ethics committees and conducted in accordance with the Declaration of Helsinki. The primary outcome was 90-day all-cause mortality.

\section{Blood Sampling and Laboratory Analyses}

Serial blood sampling was performed at baseline $(0 \mathrm{~h})$, $12,24,36,48,72,96$, and 120 hours, and plasma aliquots were stored at $-70 \mathrm{C}^{\circ}$ until assayed. All patients with available baseline plasma samples $(n=177)$ were included in this study. Creatinine, C-reactive protein, alanine aminotransferase, high-sensitivity troponin $\mathrm{T}$ (hsTnT), N-terminal pro-B-type natriuretic peptide (NT-proBNP), and GDF-15 (all assays from Roche Diagnostics) were analyzed at a central laboratory (ISLAB, Kuopio, Finland). GDF-15 levels $<1200 \mathrm{ng} / \mathrm{L}$ were considered normal (the 90th percentile in a study on healthy elderly adults). ${ }^{7,18}$ Arterial blood lactate and $\mathrm{pH}$ were analyzed locally. Estimated glomerular filtration rate (eGFR) was calculated from creatinine values using the CKD-EPI (Chronic Kidney Disease Epidemiology Collaboration) equation. ${ }^{19}$

\section{Statistical Analysis}

Descriptive data are presented as numbers (n) and percentages (\%) for categorical variables, and as mean and standard deviation (SD) or as median and interquartile range (IQR) for continuous variables, as appropriate. Patients were dichotomized according to the median baseline GDF-15 level. Between groups comparisons were performed using Chi-squared test for categorical variables, and Student's $t$ test, Mann-Whitney $U$ test, or Wilcoxon signed rank test for continuous variables, as appropriate. Correlation analyses were performed by Spearman test.

To investigate the changes in GDF-15 levels and their impact on the outcome we created a delta-variable $(\Delta \mathrm{GDF}$ $0-48 \mathrm{~h}$ ) by calculating the largest change in the biomarker level between two samples $\geq 24$ hours apart during the first 48 hours. The adequate number of samples required for calculation was available from 146 patients. We categorized the delta-variables into 3 groups regarding the change in the biomarker level 1) no change ( $\leq 30 \%$ increase or decrease), 2) $>30 \%$ increase, and 3) $>30 \%$ decrease.

Kaplan-Meier curves were used to illustrate the timing of events during follow-up between the groups and statistical comparison was performed using the log rank test. Univariate and multivariable logistic regression analyses were used to evaluate the association of GDF-15 levels with 90-day mortality. The model was adjusted with the CardShock risk score variables. ${ }^{3}$ The CardShock risk score is a 9-point risk prediction tool for in-hospital mortality consisting of seven clinical parameters that are readily available on admission (age, eGFR, blood lactate, confusion on admission, left ventricular ejection fraction [LVEF], previous myocardial infarction or coronary artery bypass grafting, and ACS etiology). Results from the logistic regression analyses are presented as odds ratios (ORs) with $95 \%$ confidence intervals (CIs). Differences in GDF-15 levels 
between survivors and non-survivors over time were analyzed with linear mixed modeling. Due to skewed distribution GDF-15 values were log-transformed to normalize the distribution and the residuals.

To assess whether GDF-15 improves discrimination beyond the CardShock risk Score, the area under the curve (AUC) of the receiver operating characteristic (ROC) curves were calculated. Youden's index was used to identify the optimal cutoff value of GDF-15 from the ROC curve. The added value of GDF-15 in the risk prediction model at different time points was assessed using the likelihood ratio test of nested models. Discrimination was also assessed by the integrated discrimination index (IDI). Improvement in clinical risk stratification was assessed by calculating net reclassification improvement (NRI) using prespecified categories of low $(0 \%-15 \%)$, intermediate $(15 \%-50 \%)$, and high $(>50 \%)$ mortality risk as previously defined for the CardShock risk score. ${ }^{3}$ A two-sided $P$ value $<.05$ was regarded as statistically significant. All statistical analyses were performed with SPSS 22.0 software (IBM, Armonk, NY) with the exception of the reclassification analyses which were performed with $\mathrm{R}$ version 3.4.1 using PredictABEL package.

\section{Results}

The characteristics of the patient population $(\mathrm{n}=177)$ are shown in Tables 1 and 2. In brief, the mean age was 66 years (SD 12), and $75 \%$ were men. Mean arterial blood pressure at enrolment was $57 \mathrm{mmHg}$ (SD 11) and median level of blood lactate was $2.7 \mathrm{mmol} / \mathrm{L}$ (IQR 1.7-5.8). ACS was the cause of CS in $80 \%$ of cases. Seventy-three patients (41\%) died during follow-up.

\section{GDF-15 Levels in Cardiogenic Shock}

The median level of GDF-15 in patients with CS was highest at baseline (GDF-15 $9647 \mathrm{ng} / \mathrm{L}$; IQR 4500-19,270), with individual values ranging from 1123 to $115,660 \mathrm{ng} / \mathrm{L}$ (levels
$<1200 \mathrm{ng} / \mathrm{L}$ are considered normal). In serial sampling, the median GDF-15 levels were $8500 \mathrm{ng} / \mathrm{L}$ (IQR 4171-17,654) at 12 hours, $6642 \mathrm{ng} / \mathrm{L}$ (IQR $3428-19,010$ ) at 24 hours, $5846 \mathrm{ng} / \mathrm{L}$ (IQR $2821-15,253$ ) at 36 hours, and $5034 \mathrm{ng} / \mathrm{L}$ (IQR 2714-12,281) at 48 hours.

Patient characteristics, medical history, and mortality of patients stratified by median GDF-15 level at baseline are shown in Table 1. The groups did not differ with regard to age, gender, body mass index, or etiology (ACS/non-ACS) of shock. However, there was a significantly higher prevalence of comorbidities, ie, diabetes mellitus and previous history of coronary artery disease, in patients with baseline GDF-15 level above median.

The clinical presentation and biochemistry at baseline stratified according to baseline GDF-15 median level are shown in Table 2. Systolic blood pressure, heart rate, and LVEF at baseline echocardiography were similar in patients with baseline GDF-15 above and below median. Patients with baseline GDF-15 above median had significantly higher levels of blood lactate, NT-proBNP, creatinine, alanine aminotransferase, and C-reactive protein, and lower arterial $\mathrm{pH}$, blood hemoglobin concentration, and eGFR.

There were significant correlations between baseline GDF-15 and baseline NT-proBNP $(\rho=0.38, P<.001)$ and lactate $(\rho=0.47, P<.001)$ with a negative correlation observed with eGFR $(\rho=-0.45, P<.001)$. Weaker correlations were observed between baseline GDF-15 and alanine aminotransferase $(\rho=0.29)$ and $C$-reactive protein ( $\rho=0.26 ; P=.001$ for both). We found no significant correlation with hsTnT either at baseline or at later time points.

\section{Baseline GDF-15 Levels and Mortality}

Higher levels of baseline GDF-15 were associated with mortality both in univariate (nGDF-15 ${ }_{0 \mathrm{~h}}$ OR $2.1 ; 95 \% \mathrm{CI}$ $1.5-2.9, P<.001)$ and multivariable $\left(\operatorname{lnGDF}-15_{0 \mathrm{~h}}\right.$ OR 1.9 ; 95\% CI 1.2-3.1, $P=.008$ ) logistic regression analyses (Fig. 1). Patients with baseline GDF-15 levels > median had

Table 1. Clinical Characteristics, In-Hospital, and 90-Day Mortality Stratified by Baseline GDF-15

\begin{tabular}{|c|c|c|c|c|}
\hline & All $(n=177)$ & GDF-15 $\leq$ Median $(n=89)$ & GDF-15 $>$ Median $(n=88)$ & $P$ Value \\
\hline Age, years (SD) & $66(12)$ & $65(12)$ & $67(13)$ & .4 \\
\hline Female, n (\%) & $45(25)$ & $20(23)$ & $25(28)$ & .4 \\
\hline BMI (SD), $\mathrm{kg} / \mathrm{m}^{2}$ & $27(4)$ & $27(4)$ & $27(4)$ & .25 \\
\hline ACS etiology, n (\%) & $142(80)$ & $71(80)$ & $71(81)$ & .9 \\
\hline STEMI, n (\%) & $119(67)$ & $61(69)$ & $58(66)$ & .7 \\
\hline Resuscitated, n (\%) & $47(27)$ & $24(27)$ & $23(26)$ & .9 \\
\hline \multicolumn{5}{|l|}{ Medical history, n (\%) } \\
\hline Hypertension & $107(60)$ & $50(56)$ & $57(65)$ & .2 \\
\hline Diabetes mellitus & $52(29)$ & $20(22)$ & $32(36)$ & .04 \\
\hline Coronary artery disease & $57(32)$ & $21(24)$ & $36(41)$ & .014 \\
\hline Prior CABG & $11(6)$ & $1(1)$ & $10(11)$ & .005 \\
\hline Heart failure & $29(16)$ & $11(12)$ & $18(20)$ & .15 \\
\hline Atrial fibrillation & $26(15)$ & $13(15)$ & $13(15)$ & 1.0 \\
\hline Renal insufficiency & $21(12)$ & $7(8)$ & $14(16)$ & .1 \\
\hline Smoking & $107(60)$ & $53(60)$ & $54(61)$ & .9 \\
\hline In-hospital mortality, n (\%) & $66(37)$ & $22(25)$ & $44(50)$ & .001 \\
\hline 90-day mortality, $n(\%)$ & $73(41)$ & $24(28)$ & $49(56)$ & $<.001$ \\
\hline
\end{tabular}

ACS, acute coronary syndrome; BMI, body mass index; CABG, coronary artery bypass surgery; SD, standard deviation; STEMI, ST-elevation myocardial infarction. 
Table 2. Clinical Presentation, Treatment, and Biochemistry on Admission

\begin{tabular}{|c|c|c|c|c|}
\hline & All $(n=177)$ & GDF-15 $\leq$ Median $(n=89)$ & GDF-15 > Median $(n=88)$ & $P$ Value \\
\hline Systolic BP; mmHg (SD) & $77(14)$ & $77(12)$ & $77(16)$ & 1.0 \\
\hline MAP; $\mathrm{mmHg}$ & $57(11)$ & $57(10)$ & $57(12)$ & .8 \\
\hline $\mathrm{HR}$, beats/min & $88(29)$ & $87(28)$ & $89(29)$ & .6 \\
\hline LVEF; \% & $33(14)$ & $35(14)$ & $31(14)$ & .10 \\
\hline Sinus rhythm, n (\%) & $127(72)$ & $73(82)$ & $54(61)$ & .001 \\
\hline Atrial fibrillation, n (\%) & $26(15)$ & $8(9)$ & $18(20)$ & .03 \\
\hline Confusion, n (\%) & $116(66)$ & $57(64)$ & $59(67)$ & .7 \\
\hline Oliguria, n (\%) & $93(53)$ & $38(43)$ & $55(63)$ & 015 \\
\hline Cold periphery, n (\%) & $169(96)$ & $85(96)$ & $84(96)$ & 1.0 \\
\hline Lactate $>2 \mathrm{mmol} / \mathrm{L}$ at inclusion, $\mathrm{n}(\%)$ & $124(70)$ & $47(53)$ & $85(96)$ & $<.001$ \\
\hline Mechanical ventilation, $\mathrm{n}(\%)$ & $97(55)$ & $43(48)$ & $54(61)$ & .08 \\
\hline \multicolumn{5}{|l|}{ Biochemistry } \\
\hline Hemoglobin; g/L & $129(23)$ & $133(24)$ & $124(21)$ & .008 \\
\hline Leukocytes; E9/L & $14.0(5.5)$ & $13.5(4.9)$ & $14.6(5.9)$ & .20 \\
\hline $\mathrm{CRP} ; \mathrm{mg} / \mathrm{L}$ & $15(4-53)$ & $7(4-40)$ & $26(5-75)$ & .01 \\
\hline Creatinine; $\mu \mathrm{mol} / \mathrm{L}$ & $103(79-140)$ & $91(68-116)$ & $125(88-157)$ & $<.001$ \\
\hline $\mathrm{eGFR} ; \mathrm{mL} / \mathrm{min} / 1.73 \mathrm{~m}^{2}$ & $63(29)$ & $73(28)$ & $53(27)$ & $<.001$ \\
\hline ALT; U/L & $45(20-93)$ & $29(17-52)$ & $82(33-152)$ & $<.001$ \\
\hline Arterial pH & $7.30(7.21-7.40)$ & $7.35(7.26-7.40)$ & $7.30(7.20-7.38)$ & .004 \\
\hline Lactate; $\mathrm{mmol} / \mathrm{L}$ & $2.7(1.7-5.8)$ & $2.1(1.3-3.7)$ & $3.7(2.3-6.7)$ & $<.001$ \\
\hline hsTnT; ng/L & $2190(393-5399)$ & $1581(347-4083)$ & $2629(441-8716)$ & .06 \\
\hline NT-proBNP; ng/L & $2581(575-9323)$ & $1360(373-6627)$ & $5029(1581-12,300)$ & $<.001$ \\
\hline GDF-15; ng/L & $9647(4500-19,270)$ & $4503(2598-6779)$ & $19,270(13,178-34,605)$ & $<.001$ \\
\hline
\end{tabular}

ALT, alanine aminotransferase; BP, blood pressure; CRP, C-reactive protein; HR, heart rate; MAP, mean arterial pressure.

a significantly higher in-hospital (50\% vs $25 \%, P=.001)$ and 90-day ( $56 \%$ vs $28 \%, P<.001$ ) mortality compared with those with GDF-15 $\leq$ median (Table 1). The Kaplan-Meier survival curves in patients stratified by median GDF-15 levels are shown in Fig. $2(\log \operatorname{rank} P<.001)$. After multivariable adjustment, baseline GDF-15 > median remained independently associated with 90-day mortality (OR 2.6; $95 \%$ CI $1.2-5.9, P=.02$ ).

\section{Serial Measurements of GDF-15 and Outcome}

GDF-15 was an independent predictor of 90-day mortality at all measured time points (Fig. 1). The AUC of GDF15 for 90-day mortality was 0.70 (95\% CI $0.62-0.77$, $P<.001$ ) at baseline, further increased at 12 hours (AUC $0.81 ; 95 \%$ CI $0.74-0.88, P<.001$ ), and remained high during the following days (Fig. 3).

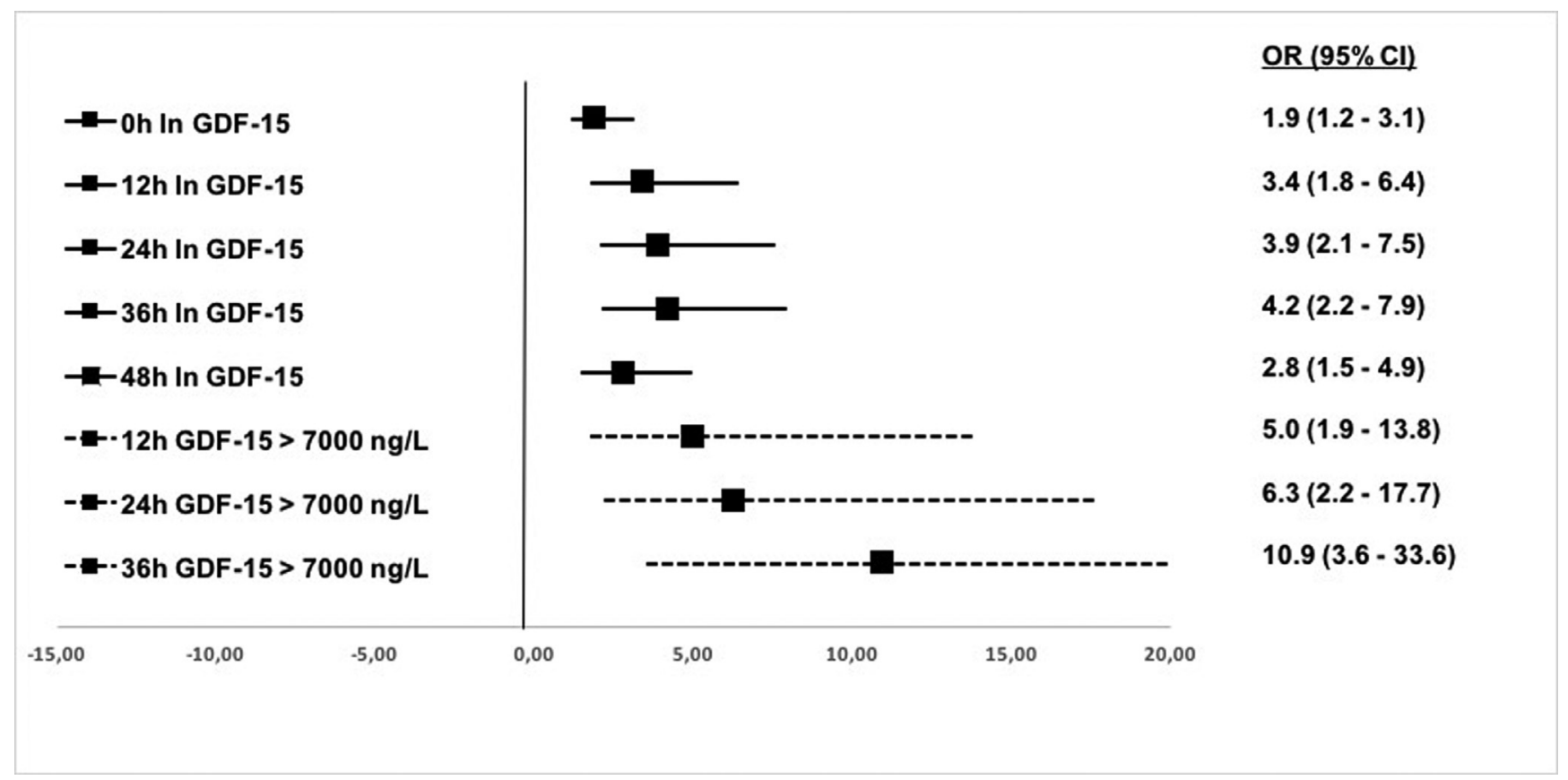

Fig. 1. Forest plot for the association of lnGDF-15 (solid line) and GDF-15 > $7000 \mathrm{ng} / \mathrm{L}$ (dashed line) at various time points with 90 -day mortality. $P<.05$ for all. The number of patients having GDF-15>7000 ng/L was $88(57 \%)$ at 12 hours, 67 (49\%) at 24 hours, and 58 $(44 \%)$ at 36 hours. 


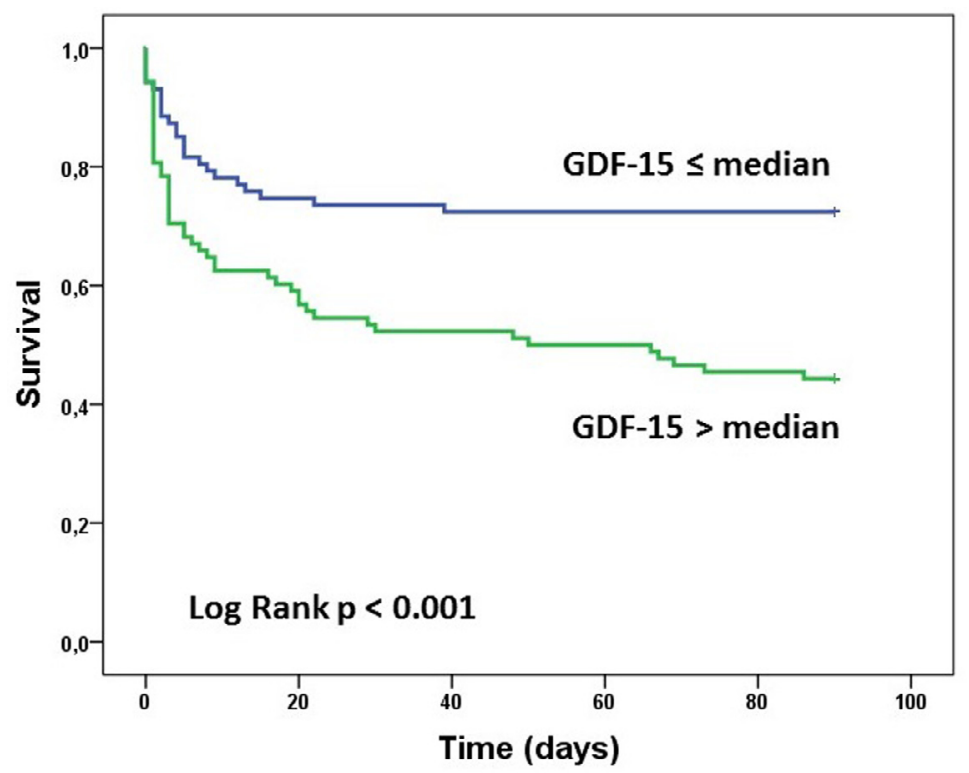

Fig. 2. Kaplan-Meier survival curves for 90-day mortality stratified by the median level of baseline GDF-15.

Serial measurement revealed that the non-survivors had significantly higher GDF-15 levels at all time points compared with the survivors (Fig. 4; $P<.001$ for between-group comparisons and $P<.001$ for all pairwise comparisons). Interestingly, there was a statistically significant decrease of the GDF-15 levels during the first 24 hours in 90-day survivors (median 6640 [IQR 3248-14,896] at baseline vs 4499 [2477-9272] $\mathrm{ng} / \mathrm{L}$ at $24 \mathrm{~h}, P<.001$ ), whereas the GDF-15 levels remained very high or even tended to increase $(12,847$ [8795-29,753] $\mathrm{ng} / \mathrm{L}$ at baseline vs 19,742 $[8815-38,240] \mathrm{ng} / \mathrm{L}$ at $24 \mathrm{~h}, P=.14)$ in patients who subsequently died (Fig. 4). Evolution of GDF over time between the survivors and the deceased at 90 days was significantly different $(P<.001$ for time-group interaction).

GDF-15 levels increased $>30 \%$ in $43(30 \%)$, decreased $>30 \%$ in $83(57 \%)$, and remained stable ( $\leq 30 \%$ increase or decrease) in $20(14 \%)$ patients during the first 48 hours. Patients with $>30 \%$ increase in GDF-15 level had worse 90-day survival than patients with stable or declining levels (Supplementary Fig. 1). However, the association with mortality of an increase in GDF-15 $>30 \%$ (compared with stable/decrease) did not reach statistical significance after

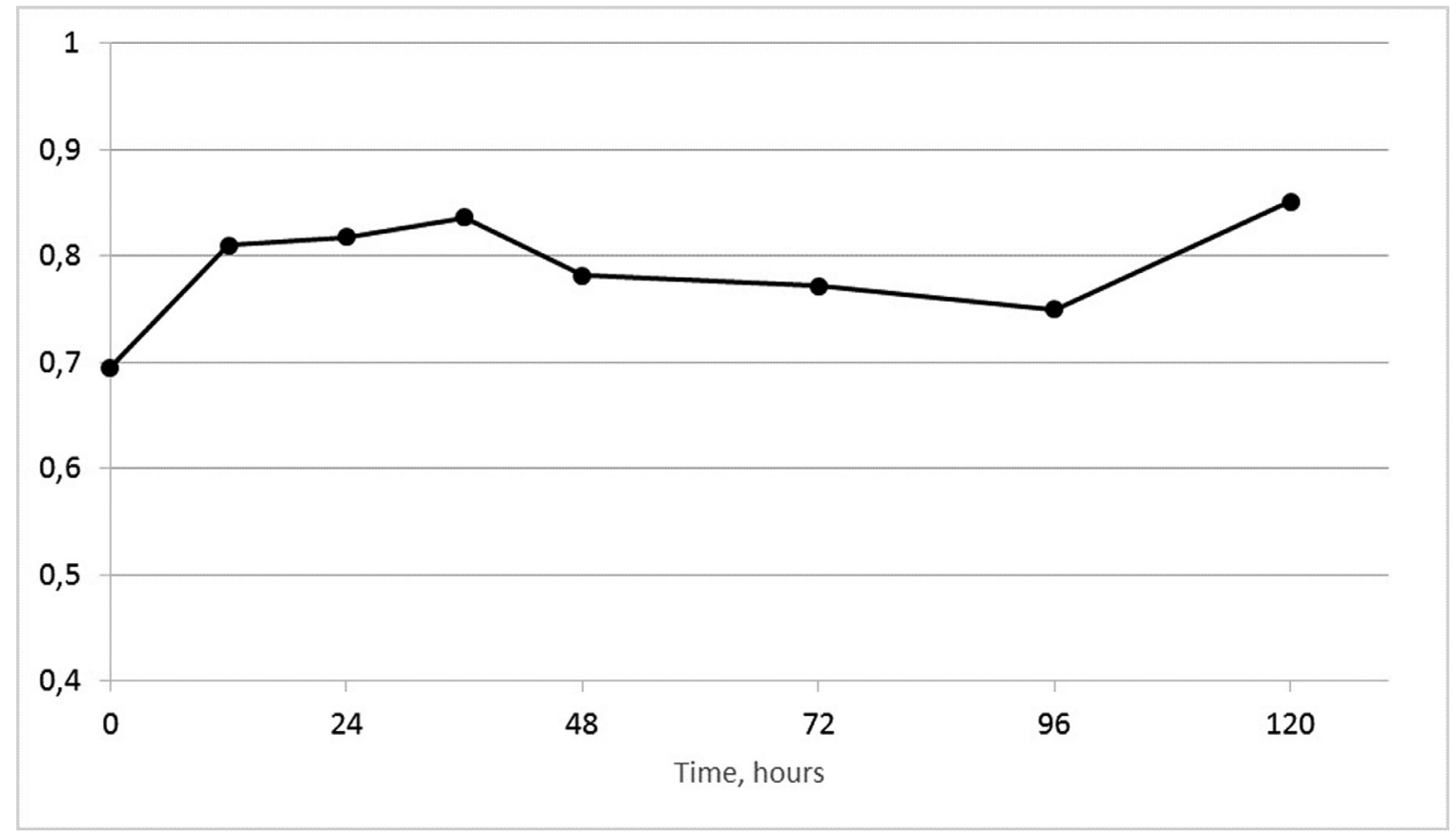

Fig. 3. AUC of GDF-15 to discriminate between 90-day survivors and non-survivors at each time point. 


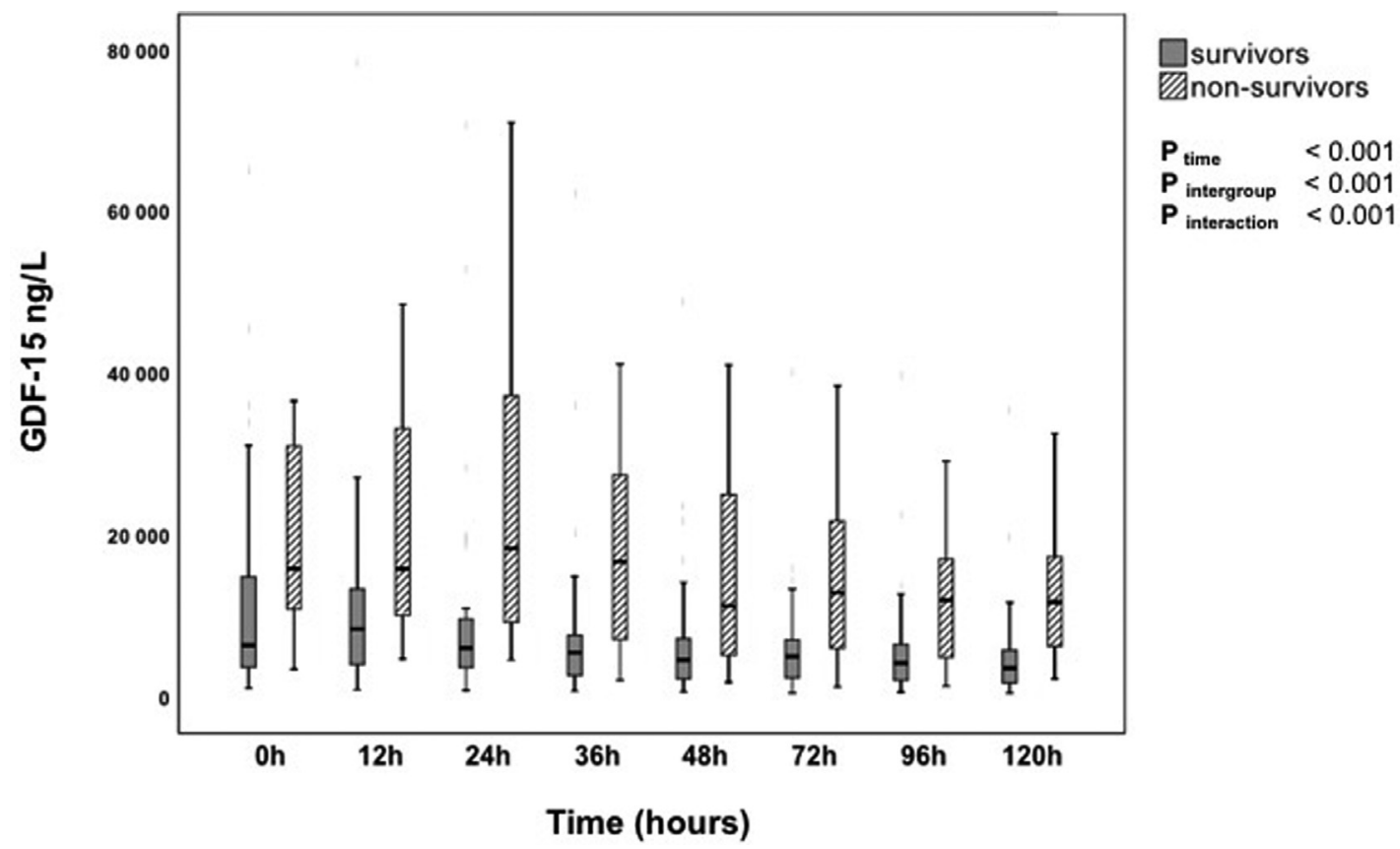

Fig. 4. GDF-15 levels $0-120$ hours in survivors and non-survivors.

adjustment for the variables in the CardShock risk score (OR 2.3 [95\% CI 0.9-5.8], $P=.07$ )

\section{GDF-15 for Risk Stratification in CS}

For early risk stratification in CS and based on the AUC values at each time-point, GDF-15 at 12 hours (GDF-15 $12 \mathrm{~h}$ ) was selected for further analyses. The GDF- $15_{12 \mathrm{~h}}$ cutoff $7000 \mathrm{ng} / \mathrm{L}$ was derived from the ROC curve (Supplementary Fig. 2) and used as a binary variable in discrimination and reclassification analyses. The adjusted OR of GDF-15 $12 \mathrm{~h}>$ $7000 \mathrm{ng} / \mathrm{L}$ for 90 -day mortality was 5.0 (95\% CI 1.9-13.8, $P<.002$ ) (Fig. 1). Adding GDF-15 $12 \mathrm{~h}>7000 \mathrm{ng} / \mathrm{L}$ to the prediction model improved discrimination compared with the CardShock risk score alone (AUC 0.85 vs AUC 0.83; $\chi^{2}=10.6, P=.001$ for comparison of nested models; and IDI 0.053 [95\% CI $0.012-0.094$ ]; $P=.01$ ). Adding GDF-15 $12 \mathrm{~h}$ $>7000 \mathrm{ng} / \mathrm{L}$ to the CardShock risk score also improved risk classification (NRI 0.18 [95\% CI $0.06-0.30 ; P=.003]$ ), especially among the survivors (Table 3; Supplementary Table 1). Sensitivity analyses were performed using the GDF-15 cutoff of $7000 \mathrm{ng} / \mathrm{L}$ also at 24 and 36 hours (Fig. 1). Clinically meaningful improvement in discrimination and reclassification was observed at any time point between 12 and 36 hours after CS detection (Table 3).

\section{Discussion}

In this prospective study with serial GDF-15 sampling in CS patients, we report 3 main findings. First, although GDF-15 levels are markedly elevated in CS already at baseline, there are marked differences in the levels and temporal trends of GDF-15 between survivors and non-survivors. Second, GDF-15 is an independent predictor of mortality in $\mathrm{CS}$, with strong predictive value early during hospitalization and throughout the hospital course. Finally, we propose a GDF-15 cutoff of $7000 \mathrm{ng} / \mathrm{L}$ that provides excellent discriminative properties for early risk stratification beyond the clinical CardShock risk score.

\section{GDF-15 Levels in CS}

In this population with CS, patients presented with extremely high levels of circulating GDF-15 at the time of detection of the shock. Virtually all patients had GDF-15 levels above the previously defined upper limit of normal

Table 3. AUC, NRI, and IDI Values for 90-Day Mortality Assessing the Capability of GDF-15 > 7000 ng/L to Improve the Discrimination and risk stratification of CardShock Risk Score (CSS) at 12, 24, and 36 hours

\begin{tabular}{|c|c|c|c|c|}
\hline & CSS & $\mathrm{CSS}+\mathrm{GDF}-15_{12 \mathrm{~h}}$ & $\mathrm{CSS}+\mathrm{GDF}-15_{24 \mathrm{~h}}$ & $\mathrm{CSS}+\mathrm{GDF}-15_{36 \mathrm{~h}}$ \\
\hline $\operatorname{AUC}(95 \% \mathrm{CI})$ & $0.83(0.77-0.89)$ & $\Delta \mathrm{AUC} 0.02 *$ & $\triangle \mathrm{AUC} 0.01 *$ & $\Delta \mathrm{AUC} 0.01^{*}$ \\
\hline NRI $(95 \%$ CI $), \%^{\dagger}$ & - & $\begin{array}{l}18.3 \\
(6.1-30.5)\end{array}$ & $\begin{array}{l}27.1 \\
(7.4-46.8)\end{array}$ & $\begin{array}{l}34.6 \\
(13.6-55.6)\end{array}$ \\
\hline IDI $(95 \% \text { CI })^{\dagger}$ & - & $\begin{array}{l}0.053 \\
(0.012-0.094)\end{array}$ & $\begin{array}{l}0.08 \\
(0.028-0.133)\end{array}$ & $\begin{array}{l}0.14 \\
(0.071-0.20)\end{array}$ \\
\hline
\end{tabular}

\footnotetext{
*P value $<.01$ for comparison of the model to CardShock risk Score alone.

${ }^{\dagger} P$ value $<.01$ for all NRI and IDI values compared with CardShock risk Score alone.
} 
(1200 ng/L) and the median GDF-15 level was two to fivefold higher than the levels previously described in patients with acute heart failure or ST-elevation myocardial infarction without CS. ${ }^{15,16,20}$ GDF-15 elevations of similar magnitude were previously found in CS patients in the biomarker substudy of the IABP-SHOCK II trial. ${ }^{21}$ Together with our results, these highly elevated levels of GDF-15 within the first 6-12 hours from onset of CS suggest a very rapid rise in the expression of GDF-15 in response to shock. The time between the onset of CS and blood sampling should therefore be taken into consideration, when interpreting GDF-15 levels in early course of CS.

In cardiogenic shock, the sources of GDF-15 are most likely to be diverse. Ischemia and reperfusion injury induce the expression of GDF-15 in cardiomyocytes during acute myocardial infarction. ${ }^{22}$ However, despite high circulating GDF-15 concentrations, cardiac mRNA and protein expression levels of GDF-15 in end-stage non-ischemic dilated cardiomyopathy were very low suggesting other sources of secretion. ${ }^{17}$ In our study, no correlation between GDF-15 and myocyte necrosis (hsTnT) was observed. In contrast, GDF-15 was associated with multiple biochemical markers of systemic hypoperfusion (hyperlactatemia, acidosis) and end-organ dysfunction (cardiac, renal, hepatic). GDF-15 is expressed in almost every tissue and strongly upregulated in acute injury and chronic stressful situations. High GDF-15 levels are known to be related to different types of organ failure (heart, liver, and kidney). Similarly to CS, very high levels of circulating GDF-15 have been detected in a small study on patients with sepsis (median GDF-15 level: 16,000 ng/L), another state of systemic hypoperfusion. ${ }^{23}$ Taken together, these results suggest GDF-15 to be a marker of systemic hypoperfusion severity and multiorgan injury and dysfunction in CS.

\section{GDF-15 Levels in Survivors and Non-Survivors}

Differences in GDF-15 levels between survivors and nonsurvivors were observed already at the time of detection of shock, in line with a previous report from the IABPSHOCK II-trial. ${ }^{21}$ Our study shows that GDF-15 levels further diverge during hospitalization between survivors and non-survivors. Our results thus suggest that stable or decreasing GDF-15 levels may be a marker of early response to treatment among patients who will survive, whereas increasing levels of GDF-15 at 24 hours despite adequate treatment are indicative of a dismal prognosis.

In addition to our study, baseline GDF-15 levels were shown to have prognostic value in CS patients also in the IABP-SHOCK II-study. ${ }^{21}$ The results from our study indicate that although baseline levels of GDF-15 associated with outcome, the prognostic capability for mortality prediction of GDF-15 is even stronger at $12-36$ hours. Considering the management during the early phase of CS (urgent revascularization, stabilization of hemodynamic, and other treatment procedures), this time frame can be regarded even more important for risk assessment and prognostication from a clinical point of view.

\section{GDF-15 for Risk Prediction in Cardiogenic Shock}

Our study demonstrates that GDF-15 possesses prognostic value beyond clinical risk prediction models for mortality in CS. There is a call for personalized medicine in general and particularly in heart failure. ${ }^{6}$ More personalized therapeutic approaches could be based on enhanced risk stratification algorithms that incorporate biomarkers. Recently, GDF-15 has been used in the $\mathrm{ABC}$ risk scores in atrial fibrillation, ${ }^{24-26}$ supporting clinical applicability of this biomarker. Personalized and precision medicine may be of particular value in the critically ill, and we believe that biomarkers may help address the persistently high mortality of patients with CS. We show that in CS, GDF-15 improves the ability to predict 90-day mortality both in terms of discrimination and reclassification in clinically useful risk categories. Although the suggested cutoff $(7000 \mathrm{ng} / \mathrm{L})$ was derived from levels measured at 12 hours, its utility was not limited by strict timing. On the contrary, GDF- 15 can be assessed in a clinically relevant time window of $12-36$ hours.

\section{Limitations}

The main limitation of our study is the lack of external validation, which should be taken into account when using the suggested cutoff. However, this is the first study to show the temporal trends of GDF-15 in CS and provides a solid basis for future studies. In addition, since the optimal cutoff value of GDF-15 was derived from the prospectively collected data in our study, in another dataset this cutoff level may overestimate the predictive capability of the biomarker causing bias. Nevertheless, our study is one of the largest cohorts of biomarker studies in CS and thus the results represent the most recent and contemporary knowledge available in the field.

\section{Conclusions}

Levels of circulating GDF-15 are very high early in CS, reflecting systemic hypoperfusion and end-organ dysfunction. Higher GDF-15 levels are independently associated with mortality, with non-survivors displaying further increase in GDF-15, whereas levels of GDF-15 in survivors decline during the first days in CS. At the proposed $7000 \mathrm{ng} / \mathrm{L}$ threshold, GDF-15 possesses the ability to add value to the CardShock risk prediction score for early discrimination (12-36 $\mathrm{h}$ after detection of shock) between survivors and non-survivors in $\mathrm{CS}$, which makes it an important biomarker for risk stratification in CS.

\section{Disclosures}

KC. Wollert holds patents and licensing contract with Roche Diagnostics, both related to GDF-15. V.-P. Harjola: Advisory board fees from Roche Diagnostics, research grant from Abbott, speaker fees from Orion, all outside the present work. J. Lassus: Speakers bureau and consultancy fees: Astra-Zeneca, Bayer, Boehringer-Ingelheim, Novartis, OrionPharma, Pfizer, Roche Diagnostics, and ViforPharma, 
all outside the present work. A. Mebazaa: lecture fees from Novartis, Orion, and Abbott, research grants from Roche, and consultant fees from Servier and Sanofi, all outside the present work. A. Kimmoun: fees for ASPEN, MSD, Gilead, and Baxter, outside the present work. A. Sionis: lecture fees from Abbott, Amgen, Astra-Zeneca, Bayer, Boeringher, Bristol-Meyers Squibb, Daiichi-Sankyo, Maquet, Menarini, Novartis, Orion-Pharma, Pfizer, Singulex, and ThermoFisher, and research grants from Novartis, Orion-Pharma, and Singulex, all outside the present work. B. Levy: lecture fees from Pulsion, Baxter, Orion, and Lilly, and consultant fees from Novartis, Orion, and Baxter, all outside the present work. K. Pulkki: Advisory board fees from Roche Diagnostics (Finland). All other authors report no relationships with industry.

\section{Acknowledgements}

We thank the CardShock study investigators in all participating hospitals.

\section{Supplementary materials}

Supplementary material associated with this article can be found in the online version at doi:10.1016/j.card fail.2019.07.003.

\section{References}

1. Hochman JS, Sleeper LA, Webb JG, et al. Early revascularization and long-term survival in cardiogenic shock complicating acute myocardial infarction. JAMA 2006;295:2511-5.

2. Thiele H, Zeymer U, Neumann FJ, et al. Intra-aortic balloon counterpulsation in acute myocardial infarction complicated by cardiogenic shock (IABP-SHOCK II): final 12 month results of a randomised, open-label trial. Lancet 2013;382: $1638-45$.

3. Harjola VP, Lassus J, Sionis A, et al. Clinical picture and risk prediction of short-term mortality in cardiogenic shock. Eur J Heart Fail 2015;17:501-9.

4. Poss J, Koster J, Fuernau G, et al. Risk stratification for patients in cardiogenic shock after acute myocardial infarction. J Am Coll Cardiol 2017;69:1913-20.

5. Chow SL, Maisel AS, Anand I, et al. Role of biomarkers for the prevention, assessment, and management of heart failure: a scientific statement from the American Heart Association. Circulation 2017;135:e1054-91.

6. Bayes-Genis A, Voors AA, Zannad F, et al. Transitioning from usual care to biomarker-based personalized and precision medicine in heart failure: call for action. Eur Heart $\mathbf{J}$ 2018:39:2793-9.

7. Wollert KC, Kempf T, Wallentin L. Growth differentiation factor 15 as a biomarker in cardiovascular disease. Clin Chem 2017;63:140-51.

8. Xu J, Kimball TR, Lorenz JN, et al. GDF15/MIC-1 functions as a protective and antihypertrophic factor released from the myocardium in association with SMAD protein activation. Circ Res 2006;98:342-50.

9. Hagstrom E, James SK, Bertilsson M, et al. Growth differentiation factor-15 level predicts major bleeding and cardiovascular events in patients with acute coronary syndromes: results from the PLATO study. Eur Heart J 2016;37:1325-33.
10. Velders MA, Wallentin L, Becker RC, et al. Biomarkers for risk stratification of patients with ST-elevation myocardial infarction treated with primary percutaneous coronary intervention: Insights from the Platelet Inhibition and Patient Outcomes trial. Am Heart J 2015;169:879-89.

11. Anand IS, Kempf T, Rector TS, et al. Serial measurement of growth-differentiation factor-15 in heart failure: relation to disease severity and prognosis in the Valsartan Heart Failure Trial. Circulation 2010;122:1387-95.

12. Sharma A, Stevens SR, Lucas J, et al. Utility of growth differentiation factor-15, a marker of oxidative stress and inflammation, in chronic heart failure: insights from the HF-ACTION study. JACC Heart Fail 2017;5:724-34.

13. Wollert KC, Kempf T, Lagerqvist B, et al. Growth differentiation factor 15 for risk stratification and selection of an invasive treatment strategy in non ST-elevation acute coronary syndrome. Circulation 2007;116:1540-8.

14. Kempf T, von Haehling S, Peter T, et al. Prognostic utility of growth differentiation factor- 15 in patients with chronic heart failure. J Am Coll Cardiol 2007;50:1054-60.

15. Cotter G, Voors AA, Prescott MF, et al. Growth differentiation factor 15 (GDF-15) in patients admitted for acute heart failure: results from the RELAX-AHF study. Eur J Heart Fail 2015; 17:1133-43.

16. Eitel I, Blase P, Adams V, et al. Growth-differentiation factor 15 as predictor of mortality in acute reperfused ST-elevation myocardial infarction: insights from cardiovascular magnetic resonance. Heart 2011;97:632-40.

17. Lok SI, Winkens B, Goldschmeding R, et al. Circulating growth differentiation factor-15 correlates with myocardial fibrosis in patients with non-ischaemic dilated cardiomyopathy and decreases rapidly after left ventricular assist device support. Eur J Heart Fail 2012;14:1249-56.

18. Kempf T, Horn-Wichmann R, Brabant G, et al. Circulating concentrations of growth-differentiation factor 15 in apparently healthy elderly individuals and patients with chronic heart failure as assessed by a new immunoradiometric sandwich assay. Clin Chem 2007;53:284-91.

19. Levey AS, Stevens LA, Schmid CH, et al. A new equation to estimate glomerular filtration rate. Ann Intern Med 2009;150: 604-12.

20. Kempf T, Bjorklund E, Olofsson S, et al. Growth-differentiation factor-15 improves risk stratification in ST-segment elevation myocardial infarction. Eur Heart J 2007;28:2858-65.

21. Fuernau G, Poenisch C, Eitel I, et al. Growth-differentiation factor 15 and osteoprotegerin in acute myocardial infarction complicated by cardiogenic shock: a biomarker substudy of the IABP-SHOCK II-trial. Eur J Heart Fail 2014;16:880-7.

22. Kempf T, Eden M, Strelau J, et al. The transforming growth factor-beta superfamily member growth-differentiation factor-15 protects the heart from ischemia/reperfusion injury. Circ Res 2006;98:351-60.

23. Mueller T, Leitner I, Egger M, Haltmayer M, Dieplinger B, et al. Association of the biomarkers soluble ST2, galectin-3 and growth-differentiation factor- 15 with heart failure and other non-cardiac diseases. Clin Chim Acta 2015;445:155-60.

24. Hijazi Z, Oldgren J, Lindback J, et al. The novel biomarkerbased $\mathrm{ABC}$ (age, biomarkers, clinical history)-bleeding risk score for patients with atrial fibrillation: a derivation and validation study. Lancet 2016;387:2302-11.

25. Hijazi Z, Oldgren J, Lindback J, et al. A biomarker-based risk score to predict death in patients with atrial fibrillation: the ABC (age, biomarkers, clinical history) death risk score. Eur Heart J 2018;39:477-85.

26. Hijazi Z, Lindback J, Alexander JH, et al. The ABC (age, biomarkers, clinical history) stroke risk score: a biomarker-based risk score for predicting stroke in atrial fibrillation. Eur Heart J 2016;37:1582-90. 